\title{
Double Top Production at Hadronic Colliders
}

\author{
Yu.P. Gouz and S.R. Slabospitsky \\ Institute for High Energy Physics, \\ Protvino, Moscow Region, 142284 Russia \\ gouz@mx. ihep.su \\ slabospitsky@mx.ihep.su
}

\begin{abstract}
We calculate the contribution of anomalous top-quark interaction with a gluon, photon, and $Z$-boson (FCNC) to $t \bar{t}$ pair production in hadronic collisions. Using the current data from Run 1 of the Tevatron we evaluate the upper limit on the the anomalous coupling of $t$-quark with gluon, $\left|\kappa_{g} / \Lambda\right| \leq 0.52 \mathrm{TeV}^{-1}$. We examine the production of double top ( $t t$ or $\overline{t t}$ ) at hadronic colliders as a result of FCNC interactions of the top quark. It is shown that the study of such reactions at LHC collider makes possible to obtain the constraint on strength of coupling at the level of $\left|\kappa_{g} / \Lambda\right| \leq 0.09 \mathrm{TeV}^{-1}$.
\end{abstract}

July 3, 2021 


\section{Introduction}

After the discovery of the top-quark at FNAL collider [1], the new direction appeared in search for new physics beyond the Standard Model (SM). As the most massive quark in the SM, the $t$-quark is naturally considered to be more sensitive to new physics than lighter fermions. Therefore, precision measurements of the top quark properties and its production mechanisms provide unique possibility to obtain information on new physics.

There are numerous speculations on possible new physics manifestations in the top quark sector. Of special interest is the study of top quark anomalous interactions due to flavour-changing neutral currents (FCNC) (see [4, 6, 7, 8, 9, 10, 11, 12]).

Indeed, the absence of elementary FCNC vertices in SM requires the consideration of loop contribution, which may generate the effective $t \rightarrow c(u)$ transitions [2]. As a result, the top quark decays via FCNC are suppressed at the level of $10^{-10}$ to $10^{-12}$, which makes their observation absolutely impossible.

Various extensions of SM lead to huge enhancement of such transitions. For example, one can compare the branching ratios of FCNC decays of the top quarks evaluating in the SM [2] and in MSSM [3]:

$$
\begin{array}{rcc} 
& \text { SM } & \text { MSSM } \\
\operatorname{Br}(t \rightarrow c g) & \approx 10^{-10} & 10^{-6} \\
\operatorname{Br}(t \rightarrow c \gamma) & \approx 10^{-12} & 10^{-8} \\
\operatorname{Br}(t \rightarrow c Z) & \approx 10^{-12} & 10^{-8}
\end{array}
$$

Therefore, any observation of FCNC decays would be an indication of a new physics beyond SM.

The strategy of searching for anomalous top quark couplings consists of three complementary approaches: (i) obtain indirect bounds from low energy processes in which top quark anomalous couplings can enter via loop processes, or from the bound on $t \rightarrow b W$ which puts limits on other decay modes of the top, (ii) direct searches at high energies for the effects of the anomalous couplings in top quark rare decays, and (iii) the study of the processes of top quark production via non-SM mechanisms.

The search for rare top decays are extensively considered (see, for example, [6, 7]). The experimental search for these decays was done by CDF 
collaboration [13]. One of the possibility for study of the direct top production is considered in [8]. In this scenario, a up (or charm) quark and a gluon from the colliding hadrons combine immediately to form an s-channel top quark, namely $u(c)+g \rightarrow t$. The consideration of other anomalous processes of the top production can be found in [9, 12].

In this note we examine a manifestation of anomalous (FCNC)top interaction in the process of top-antitop production:

$$
p(\bar{p}) p \rightarrow t \bar{t} X
$$

as well as in the process of double top (or antitop) production:

$$
p(\bar{p}) p \rightarrow t t X
$$

The article is organised as follows. The anomalous FCNC interaction of $t$ quark is considered in Section 2. The available constraints on anomalous couplings are considered in Section 3. In Section 4 we evaluate the upper limit on FCNC top interaction with a gluon from Run 1 of the Tevatron. The estimate for possible constraint on the same coupling, which may be reached at LHC collider, is given in Section 5. Section 6 presents general characteristics of the process of double ( $t t$ or $\overline{t t}$ ) top production in hadronic collisions. The study of this reaction and possible background processes are presented in Section 7. The main results are summarized in Conclusion.

\section{Anomalous $\bar{t} c$ Vertices}

Following [5], the vertices of the FCNC transitions $t \rightarrow g c, t \rightarrow \gamma c$, and $t \rightarrow Z c$ can be written as follows:

$$
\begin{aligned}
t g c & \Rightarrow g_{s} \frac{\kappa_{g}}{\Lambda} \bar{t} \sigma_{\mu \nu}\left[g_{L} P_{L}+g_{R} P_{R}\right] t^{a} c G^{a \mu \nu}, \\
t \gamma c & \Rightarrow e \frac{\kappa_{\gamma}}{\Lambda} \bar{t} \sigma_{\mu \nu}\left[\gamma_{L} P_{L}+\gamma_{R} P_{R}\right] c F^{\mu \nu}, \\
t Z c & \Rightarrow \kappa_{z} \frac{e}{\sin 2 \vartheta_{W}} \bar{t} \gamma_{\mu}\left[z_{L} P_{L}+z_{R} P_{R}\right] c Z^{\mu},
\end{aligned}
$$

where $\Lambda$ is the new physics cutoff, $\vartheta_{W}$ is the Weinberg angle, $P_{L, R}=\frac{1}{2}\left(1 \mp \gamma^{5}\right)$, $\sigma^{\mu \nu}=\frac{1}{2}\left(\gamma^{\mu} \gamma^{\nu}-\gamma^{\nu} \gamma^{\mu}\right), \kappa_{g}, \kappa_{\gamma}$, and $\kappa_{z}$ define the strength of the anomalous couplings for the currents with a gluon $\left(\kappa_{g}\right)$, photon $\left(\kappa_{\gamma}\right)$, and $Z$ boson $\left(\kappa_{z}\right)$, 
respectively. The magnitudes of left and right components of the currents are denoted by $g_{L, R}, \gamma_{L, R}, z_{L, R}$. They obey the obvious constraints:

$$
g_{L}^{2}+g_{R}^{2}=1, \quad \gamma_{L}^{2}+\gamma_{R}^{2}=1, \quad z_{L}^{2}+z_{R}^{2}=1
$$

We also assume that $\operatorname{Im} \kappa_{g}=\operatorname{Im} \kappa_{\gamma}=\operatorname{Im} \kappa_{z}=\operatorname{Im} \gamma_{i}=\operatorname{Im} g_{i}=\operatorname{Im} z_{i}=0$, see [5, 6].

Generally speaking, to avoid unitarity violation, the anomalous couplings $\kappa$ should depend on momentum transfer $q^{2}$. For example, this dependence may have a form-factor-like behaviour

$$
\frac{\kappa_{g, \gamma}^{2}}{\Lambda^{2}} \Rightarrow \frac{\kappa_{g, \gamma}^{2}}{\Lambda^{2}\left(1+\frac{q^{2}}{\Lambda^{2}}\right)}, \quad \kappa_{z}^{2} \Rightarrow \frac{\kappa_{z}^{2}}{1+\frac{q^{2}}{\Lambda^{2}}} .
$$

However, such a dependence should be essential at very high values of momentum transfer $(\geq 1 \mathrm{TeV})$, and we ignore such dependence in the present article.

Using the expressions for the vertices (3 5) we find the equations for the corresponding widths (see also [6, 8, 10]):

$$
\begin{aligned}
\Gamma(t \rightarrow c g) & =\frac{4}{3} \alpha_{s} m_{t}^{3}\left(\frac{\kappa_{g}^{2}}{\Lambda^{2}}\right), \\
\Gamma(t \rightarrow c \gamma) & =\alpha m_{t}^{3}\left(\frac{\kappa_{\gamma}^{2}}{\Lambda^{2}}\right), \\
\Gamma(t \rightarrow c Z) & =\frac{\alpha \kappa_{z}^{2}}{8 \sin ^{2} 2 \vartheta_{W} M_{Z}^{2}} m_{t}^{3}\left(1-\frac{M_{Z}^{2}}{m_{t}^{2}}\right)^{2}\left(1+2 \frac{M_{Z}^{2}}{m_{t}^{2}}\right),
\end{aligned}
$$

where $\alpha$ and $\alpha_{s}$ are the QED and QCD coupling constants, respectively, and $M_{Z}$ is the mass of $Z$ boson.

In what follows we set the masses of the light quark ( $c$ or $u$ ) to zero, $m_{c}=m_{u}=0$, and

$$
m_{t}=175 \mathrm{GeV} \text {. }
$$

We also assume that the anomalous couplings of top quark with $c$ and $u$ quarks are equal in magnitude.

The corresponding values of the widths and branching ratios for different decay channels are given in the Table 1. 


\section{Current Constraints on $\left|\kappa_{g}\right|$ and $\left|\kappa_{\gamma}\right|$}

The analysis of low energy processes (such as $K_{L} \rightarrow \mu^{+} \mu^{-}, K_{L}-K_{S}$ mass difference, $B^{0}-\bar{B}^{0}$ mixing, $B \rightarrow l^{+} l^{-} X, b \rightarrow s \gamma$, etc. (see, for example [6, 7] and references therein) results in the following constraints:

$$
\begin{aligned}
& \left|\kappa_{g} / \Lambda\right|<0.95 \mathrm{TeV}^{-1} \\
& \left|\kappa_{\gamma} / \Lambda\right|<0.28 \mathrm{TeV}^{-1} \\
& \left|\kappa_{z}\right|<0.29
\end{aligned}
$$

CDF collaboration performs the search for decays $t \rightarrow \gamma c(u)$ and $t \rightarrow$ $Z c(u)$ at FNAL collider in the reaction of $\bar{p} p \rightarrow \bar{t} t X$ at the energy of $\sqrt{s}=1.8 \mathrm{TeV}$. They obtained the upper limits on branching fractions for the decays $t \rightarrow \gamma c(u)$ and $t \rightarrow Z c(u)$ [13] as follows:

$$
\begin{array}{lll}
\mathrm{Br}(t \rightarrow c \gamma)+\mathrm{Br}(t \rightarrow u \gamma)<3.2 \% & (95 \% & \mathrm{CL}), \\
\mathrm{Br}(t \rightarrow c Z)+\operatorname{Br}(t \rightarrow u Z)<33 \% & (95 \% & \mathrm{CL}) .
\end{array}
$$

Using the equations (8) and (9), one can easily evaluate the upper limits on the constants of $\kappa_{\gamma}$ and $\kappa_{z}$ (at $\left.m_{t}=175 \mathrm{GeV}\right)$ from the experimental constraints (10) and (11):

$$
\begin{aligned}
& \left|\kappa_{\gamma} / \Lambda\right|<0.77 \mathrm{TeV}^{-1} \\
& \left|\kappa_{z}\right|<0.74 .
\end{aligned}
$$

Additional experimental constraints may be obtained from the study of the reaction $e^{+} e^{-}$annihilation [10]

$$
e^{+} e^{-} \rightarrow \gamma^{*}\left(Z^{*}\right) \rightarrow t \bar{c}
$$

Starting from Summer 1997, the $e^{+} e^{-}$collider LEP-2 operates at the energy

of $\sqrt{s}=184 \mathrm{GeV}$ (1997) and $189 \mathrm{GeV}$ (1998). At this in the reaction (13) becomes kinematically possible, and therefore it will be possible to improve significantly the upper limits on the anomalous couplings of top interaction (see [10] for details).

\section{Constraint on $\left|\kappa_{g}\right|$ from Run 1 of Tevatron}

In this section we consider what limit on anomalous coupling $\left|\kappa_{g}\right|$ can be derived from the available data on the cross section for $t \bar{t}$ pair production at Run 1 of the Tevatron. 
In order to obtain a constraint on $\kappa_{g}$, we analyse the contribution of anomalous top interaction into $t \bar{t}$ production at the FNAL collider.

Indeed, both CDF and D collaborations measured the top quarks production in $b W$ final states. For the case, when any non-SM top interaction are very small, one has:

$$
\operatorname{Br}(t \rightarrow W b) \approx 1 \quad \text { and } \quad \sigma_{t \bar{t}}^{\exp }=\sigma_{t \bar{t}}^{\mathrm{SM}} .
$$

However, for large values of the anomalous couplings the branching ratio to $W b$ final state may be substantially smaller than $100 \%$ (see Table 1 ). At the same time, there appeared new subprocesses leading to additional sources of the $t \bar{t}$ production. In this case the observed cross section (in the $b \bar{b} W^{+} W^{-}$ final state) should be equal to:

$$
\sigma\left(b W^{+} \bar{b} W^{-}\right)=\operatorname{Br}^{2}(t \rightarrow b W) \times \sigma(\mathrm{SM}+\text { New Physics }) .
$$

Therefore, the difference between observed cross section for $t \bar{t}$ pair production and that calculated within (SM+New Physics) framework may be used to estimate upper limits on strength of anomalous coupling.

To obtain the corresponding constraints on the anomalous couplings we require that

$$
\left|\sigma_{t \bar{t}}^{\exp }-\sigma\left(b W^{+} \bar{b} W^{-}\right)\right| \leq 2 \Delta
$$

where $\Delta$ is an error consisting of both experimental and theoretical uncertainties.

In the lowest order of perturbative QCD the $t \bar{t}$ production proceeds via quark-antiquark and gluon-gluon annihilation (see Fig.1a and 1c). The anomalous top interactions lead to the appearance of new processes in which $t \bar{t}$ can be produced. The lowest order diagrams corresponding to these processes are presented in Fig.1b and 1d.

In further analysis we assume that other non-standard decay channels of the top are negligible.

The production cross section measured by the CDF collaboration with an integrated luminosity of $110 \mathrm{pb}^{-1}$ is $\sigma=7.6_{-1.5}^{+1.8} \mathrm{pb}$ for $m_{t}=175 \mathrm{GeV}$ [14], which combines dilepton, lepton + jets and all-hadronic channels. The $\mathrm{D} \emptyset$ collaboration gives $\sigma=5.9 \pm 1.7 \mathrm{pb}$ for $m_{t}=172 \mathrm{GeV}$ [15]. In our analysis we use the combined result [16] of

$$
\sigma_{t \bar{t}}^{\exp }=6.7 \pm 1.3 \mathrm{pb} .
$$


For the theoretical cross section in the SM, we adopt the most complete result currently available [17, which includes soft-gluon summation up to the next-to-leading logarithmic order:

$$
\sigma_{t \bar{t}}^{\mathrm{SM}}=5.06_{-0.36}^{+0.13} \mathrm{pb}
$$

for $m_{t}=175 \mathrm{GeV}$ at $\sqrt{s}=1.8 \mathrm{TeV}$.

To make a comparison of the measured $\left(\sigma_{t \bar{t}}^{\exp }\right)$ and calculated cross section $\left(\sigma\left(b W^{+} \bar{b} W^{-}\right)\right)$, one should take into account the present uncertainty of the top quark mass [16], which affects both the experimental and theoretical cross sections. Shifting $m_{t}$ by $\pm 5 \mathrm{GeV}$ changes the theoretical cross section by about $\pm 15 \%$. The measured cross section also changes with $m_{t}$ in the same direction, but the dependence is weaker.

Combining all these uncertainties, the possible new physics contribution to the cross section is found to be

$$
\Delta=\sqrt{\Delta \sigma_{\exp }^{2}+\Delta \sigma_{\mathrm{th}}^{2}} \approx 1.67 \mathrm{pb},
$$

where we set 14, 15 $\Delta \sigma_{\exp }=1.3 \mathrm{pb}($ see $(16)$ ), and 17

$$
\Delta \sigma_{\mathrm{th}}=(0.15 \div 0.20) \sigma_{t \bar{t}}^{\mathrm{SM}} \text {. }
$$

In our case the inequality (15) depends essentially on two parameters, $\kappa_{g}$ and $\kappa_{z}$. The contribution due to a photon exchange $\left(\sim \kappa_{\gamma}\right)$ is very small.

Bearing in mind different notations and normalizations used in the literature, we present the evaluated constraints on anomalous constants in terms of constraints on corresponding branching ratios for top quark decays.

The resulting constraints on the branching fractions $\operatorname{Br}(t \rightarrow g(c+u))$ and $\operatorname{Br}(t \rightarrow Z(c+u))$ are shown in Fig.2. The dashed band in this figure corresponds to two choices of $\operatorname{Br}(t \rightarrow \gamma(c+u))=0 \%$ and $\operatorname{Br}(t \rightarrow \gamma(c+$ $u))=3.2 \%$. The maximum allowed values of these branching ratios are the following:

$$
\begin{aligned}
\operatorname{Br}(t \rightarrow g(c+u)) & \leq 20 \% \quad(\text { at } \quad \operatorname{Br}(t \rightarrow Z(c+u))=0) \\
\operatorname{Br}(t \rightarrow Z(c+u)) & \leq 32 \% \quad(\text { at } \operatorname{Br}(t \rightarrow g(c+u))=0)
\end{aligned}
$$

These constraints correspond to the maximum allowed value of $\kappa_{g}$ (at $\left.\kappa_{z} \rightarrow 0\right)$ as follows:

$$
\left|\kappa_{g} / \Lambda\right| \leq 0.52 \mathrm{TeV}^{-1}
$$


We conclude that the variation of $\sigma_{t \bar{t}}$ within given uncertainty $2 \Delta$ does not allow to improve the upper limit on branching fraction to $Z$-boson channel. On the other hand, from Run 1 data we obtain the lowest current constraint on FCNC top quark interaction with a gluon.

The differential distributions of top quarks produced in the reaction

$$
\bar{p} p \rightarrow t \bar{t} X
$$

at $\sqrt{s}=1.8 \mathrm{TeV}$ are shown in Fig.3. It is seen from these pictures that at the FNAL collider energies the FCNC interaction gives practically the same form of the differential distributions of the top quarks like QCD calculations.

\section{$5 \quad$ Possible Constraints on $\left|\kappa_{g}\right|$ and $\left|\kappa_{z}\right|$ from $t \bar{t}$ Production at LHC}

Contrary to the case of the FNAL collider, the contribution of FCNC top quark interaction leads to significant modification of differential spectra at LHC energies. Fig.4 presents the corresponding distributions of $t$ quarks produced in the reaction

$$
p p \rightarrow t \bar{t} X
$$

at $\sqrt{s}=14 \mathrm{TeV}$. In fact, the FCNC interaction results in significant increase of $t \bar{t}$ production with high invariant mass $M_{t \bar{t}}$ (see Fig.4). Therefore, the observation of such deviation from QCD prediction could be considered as a signal from anomalous top quark interaction'].

At the same time, the value of this effect depends on the magnitudes of the anomalous couplings, $\kappa_{q}, \kappa_{z}$, and $\kappa_{\gamma}$. Indeed, for anomalous coupling values about $\sim 10^{-2} \div 10^{-3}$ this effect becomes very small. Therefore, the reaction of $t \bar{t}$ production may be not a good method for search for FCNC top quark interaction,

Nevertheless, we estimate the possible limits on the anomalous coupling at LHC by using the same inequality (15). For the difference of the cross

\footnotetext{
${ }^{1}$ To be more precise, the shape of these distributions is determined by the point-like behavior of the effective couplings $\kappa$ (see explanation of the equation (6)) ). However, for very high value of $\Lambda$ (say, $\Lambda \geq$ few $\mathrm{TeV}$ ) we can ignore $q^{2}$-dependence of $\kappa$, and our calculated distributions remain the same.
} 
sections calculated in $\mathrm{SM}$ and $\mathrm{SM}+\mathrm{FCNC}$ frameworks (the $\Delta$ parameter) we use:

$$
\Delta_{\mathrm{LHC}}=\sqrt{0.15_{t h}^{2}+0.15_{\text {exp }}^{2}} \times \sigma_{t \bar{t}}^{\mathrm{SM}} \approx 175 \mathrm{pb}
$$

The corresponding constraint on $\operatorname{Br}(t \rightarrow g(c+u))$ (versus $\operatorname{Br}(t \rightarrow Z(c+$ $u))$ ) is shown in Fig.5. The maximum allowed value of this branching ratio is about

$$
\operatorname{Br}(t \rightarrow g(c+u)) \leq 27 \%
$$

The corresponding maximum allowed value of $\kappa_{g}\left(\right.$ at $\left.\kappa_{z} \rightarrow 0\right)$ is:

$$
\left|\kappa_{g} / \Lambda\right| \leq 0.64 \mathrm{TeV}^{-1}
$$

Note that this constraint on strength of coupling with a gluon is practically the same as that evaluated from the data of Run 1 of the Tevatron. Naturally, the obtained values of the upper limits (22) and (23) are determined by our assumption on $\Delta_{\mathrm{LHC}}$ form (21).

\section{Double Top Production at Hadronic Col- liders}

The FCNC processes result in appearance of the new processes of the top quarks production:

$$
\begin{aligned}
g u(c) & \rightarrow t \\
g u(c) & \rightarrow t Z(\gamma, g) \\
q \bar{q} & \rightarrow t \bar{c}(\bar{u}) \\
g g & \rightarrow t \bar{c}(\bar{u}) \\
\ldots &
\end{aligned}
$$

These are the processes of direct top production, considered, for example, in [8, 9]. Note, however, that all these processes have huge QCD-background from $W+n(j e t)$ production, as well as from $t \bar{t}$ production. 
In this article we examine the process which has no background from usual $t \bar{t}$ production. Namely, we investigate the process of double top (or antitop) production:

$$
p p \rightarrow t t(\bar{t} \bar{t}) X .
$$

Many processes may result in inclusive production of $t t(\overline{t t})$ pair in hadronhadron collisions. For example:

$$
\begin{array}{ll}
\text { - } & g g \rightarrow t \bar{t} t \bar{t} \\
\text { - } & t t \rightarrow t t \\
\text { - } & d d \rightarrow t W^{-} t W^{-} \\
\text {- } & u \bar{d} \rightarrow g^{*} W^{*} \rightarrow t \bar{t} t \bar{b} \\
\text { etc } &
\end{array}
$$

However, most of these reactions are highly suppressed by higher orders of strong and/or electro-weak coupling constants. Other suppressions result from specific kinematics of the production processes. The non-top final particles should be produced in 'invisible' region for detector, i.e. they should escape into very forward/backward direction.

On the other hand, the $t t(\overline{t t})$ pair from the reaction $(\overline{24})$ may be produced in a simple $2 \rightarrow 2$ subprocess with FCNC interaction. The corresponding diagrams describing $t t$ production via anomalous top quark interaction are shown in Fig.6.

Naturally, to distinguish this process from conventional $t \bar{t}$ pair production one should require that both $t$-quarks decay semileptonically. In other words, we consider the reaction with two like-sign isolated leptons in the final state.

We notice two advantages of the reaction (24):

- it has no background from usual $t \bar{t}$ production (because $t \bar{t}$ pair decays into $l^{+} l^{-}$pair)

- to detect such a reaction one can apply the same methods as for the case of $t \bar{t}$ production.

The total cross sections for the reaction (24) at $\sqrt{s}=14 \mathrm{TeV}, m_{t}=$ $175 \mathrm{GeV}$, and $\kappa_{g}=\kappa_{\gamma}=\kappa_{z}=1$ are equal to:

$$
\begin{aligned}
\sigma(t t) & \approx 480 \mathrm{pb} \\
\sigma(\overline{t t}) & \approx 16 \mathrm{pb} .
\end{aligned}
$$


Note that for this choice of the anomalous coupling the relative contributions due to gluon, photon, and $Z$-boson exchange are

$$
g: \gamma: Z \approx 70: 5: 20
$$

These values of the cross sections correspond to the following number of events (for the total integrated luminosity of $10^{5} \mathrm{pb}^{-1}$ and $100 \%$ efficiency of the isolated lepton detection):

$$
\begin{aligned}
& \mathrm{N}\left(l^{+} l^{+} \text {jet jet }\right)_{l=\mu, e} \approx 1.9 \cdot 10^{6}, \\
& \mathrm{~N}\left(l^{-} l^{-} \text {jet jet }\right)_{l=\mu, e} \approx 6.4 \cdot 10^{5} .
\end{aligned}
$$

Requiring the observation of at least one event with $l^{+} l^{+}+2$-jets in the final state, we can estimate the value of the anomalous coupling $\kappa_{g}$ which could be reached at LHC (at $\left.\kappa_{\gamma}=\kappa_{z}=0\right)$ :

$$
\left|\kappa_{g} / \Lambda\right| \leq 2.7 \cdot 10^{-2} \mathrm{TeV}^{-1} .
$$

This value is comparable with that obtained from the study of the reaction of the direct top production in the subprocess of $g u \rightarrow c$ (see [8]).

The differential distributions for the $t$ quark from the reaction (24) are shown in the Fig.7. Like for the case of $t \bar{t}$ production, the FCNC interaction results in significant increasing of $t t$ production with a high invariant mass of $M_{t t}$ (see Fig.7).

\section{Signal and Background Calculations}

Previous estimate was obtained in pure 'ideal' case (i.e. $100 \%$ efficiency for detection of leptons and jets and we assume no background process). Now we proceed to more 'realistic' estimates.

The major sources of background to the $t t$ production are

$$
\begin{aligned}
q q^{\prime} & \rightarrow t \bar{t} W^{ \pm} \\
q q & \rightarrow W^{ \pm} q^{\prime} W^{ \pm} q^{\prime}
\end{aligned}
$$

The typical diagrams for each background process are shown in Fig.8 (see Fig.8a for the process (25) and Fig.8b for the process (26), respectively). 
The total cross sections for these background processes are (at $\sqrt{s}=$ $14 \mathrm{TeV})$

$$
\begin{array}{lll}
\sigma\left(W^{+} t \bar{t}\right) & =0.5 \mathrm{pb}, \quad \sigma\left(W^{-} t \bar{t}\right) & =0.24 \mathrm{pb} \\
\sigma\left(W^{+} W^{+} q q\right) & =0.5 \mathrm{pb}, \quad \sigma\left(W^{-} W^{-} q q\right)=0.23 \mathrm{pb} .
\end{array}
$$

In order to simulate the detector effects in identifying the signal, we made a series of standard cuts on the transverse momentum (energy) $p_{T}\left(E_{T}\right)$, pseudorapidity $\eta$, and the jet-lepton separation $\Delta R$. We call them the 'standard' cuts:

- $p_{\top}^{l}>15 \mathrm{GeV},\left|\eta_{l}\right|<2.5$

- $\Delta R_{l j}>0.4$

- $E_{\top}^{j e t}>20 \mathrm{GeV},\left|\eta_{j}\right|<2.5$

- two isolated like-sign leptons

We take a standard b-tagging with the efficiencies for $b$-quarks, charmed quarks, and light partons ( $u, d, s$ quarks and gluon) as follows:

$$
\epsilon_{b}=36 \%, \epsilon_{c}=10 \%, \epsilon_{u, d, s}=1 \% .
$$

We use the PYTHIA 5.7 + JETSET 7.4 Monte Carlo with addition of the routines calculating the signal and background processes. The result of the application of 'standard' cuts is shown in the Table 2. For the sake of comparison, we started from equal number of events $\left(N_{\text {start }}=10^{4}\right)$ for each reaction, (24), (25), and (26).

The resulting distributions are shown in Fig.9-12. From these figures one may deduce the difference in the signal and background.

First, in the signal the average number of jets is smaller than in background from $t \bar{t} W$ (see Fig.9). Both signal and background have jets with $E_{\top}^{\text {jet }}$ greater than $40-60 \mathrm{GeV}$ (see Fig.10). The invariant mass of the isolated charged lepton and jets from 'true' combinations (i.e. those coming from the decay of the same top quark) can not exceed the top mass (compare Figs. 11a and 11b). The invariant mass of the two isolated charged leptons and two jets from the signal is much higher than that for background events (see Fig.12). 
In order to further enhance the signal with respect to background we impose the following additional cuts:

"two jets" we require exactly two jets with " $E_{T}^{\text {jet }}>20 \mathrm{GeV}$;

" $E_{\top}$ " the energy of these two jets should be greater than $40 \mathrm{GeV}$, and at least one jet should be tagged as a $b$-jet;

" $M(l j)$ " at least in one combination of the lepton+jet pairs both pairs should have invariant masses smaller than $160 \mathrm{GeV}$ (i.e. $M\left(l_{1} j_{1}\right)<160 \mathrm{GeV}$ and $M\left(l_{2} j_{2}\right)<160 \mathrm{GeV}$ );

"M(ll $l j j)$ " the invariant mass of two charged leptons and two jets should be greater than $500 \mathrm{GeV}$.

The result of application of all these additional cuts (step-by-step) are shown in the Table 2. One may deduce that applying all these cuts provide almost complete suppression of the background (even at high luminosity option).

\section{Discussion and Summary}

We may use the results of the signal and background calculations to determine the maximum allowed value of $\kappa_{g} / \Lambda$ that could be reached at LHC collider. We use the following criterion

$$
N_{S} \geq 3 \sqrt{N_{S}+N_{B}}
$$

where $N_{S}$ and $N_{B}$ are the number of signal and background events, respectively. This criterion corresponds approximately to the $95 \%$ confidence level.

For the total luminosity of $\int \mathcal{L} d t=100 \mathrm{fb}^{-1}$ we have $N_{B}=2.7$ (for $\mu \mu$ mode) and $N_{B}=10.8$ (for $l l=\mu \mu+\mu e+e e$ ) (see Table 2). As a result we obtain (for $\kappa_{z}=\kappa_{\gamma}=0$ )

$$
\begin{aligned}
\left|\kappa_{g} / \Lambda\right| & \leq 0.116_{\mu \mu}\left(0.091_{l l}\right) \mathrm{TeV}^{-1}, \\
\operatorname{Br}(t \rightarrow g(c+u)) & \leq 1.2 \times 10^{-2}{ }_{\mu \mu}\left(7.4 \times 10^{-3}{ }_{l l}\right) .
\end{aligned}
$$

In summary, we calculate the contribution for $t \bar{t}$ pair production resulted from the anomalous top quark interaction (FCNC). From the analysis of the data from Run 1 of the Tevatron we deduce the lowest current limit on the anomalous coupling $\kappa_{g} / \Lambda<0.52 \mathrm{TeV}^{-1}$. 
We investigate the double top-quark production at LHC collider via the chromo-magnetic flavor-changing neutral current couplings $\bar{t} c g$ and $\bar{t} u g$. We

find that the strength of the anomalous coupling $\bar{t} q g$ may be probed to $\kappa_{g} / \Lambda=0.09 \mathrm{TeV}^{-1}$ at the LHC with $100 \mathrm{fb}^{-1}$ of data at $\sqrt{s}=14 \mathrm{TeV}$. Assuming $\kappa_{g} \equiv 1$, the scale of new physics $\Lambda$ can be probed to $11 \mathrm{TeV}$ at the LHC.

\section{Acknowledgements}

We thank to A. Arbuzov, M. Cobal, F. Froidevaux, M. Mangano, R. Mekhdiev, J. Parsons, V. Obraztsov, A. Zaitsev, and O. Yushchenko for fruitful discussions. This work was supported, in part, by Russian Foundation for Basic Research, projects no. 96-15-96575. 


\section{References}

[1] CDF Collaboration, Abe F. et al., // Phys. Rev. Lett. 74 (1995) 2626;

$D \emptyset$ Collaboration, Abachi S. et al., // Phys. Rev. Lett. 74 (1995) 2632.

[2] Grzadkowski B., Gunion J.F., and Krawczyk P. // Phys. Lett. B268 (1991) 106;

Eilam G., Hewett J.L, and Soni A. // Phys. Rev. D44 (1991) 1473;

Luke M. and Savage M.J. // Phys. Lett. B307 (1993) 387.

[3] Li C.S, Oakes R.J., and Yang J.M. // Phys. Rev. D49 (1994) 293;

Yang J.M. and Li C.S // Phys. Rev. D49 (1994) 3412;

Couture G., Hamzaoui C., and Kønig H. // Phys. Rev. D52 (1995) 1713;

Couture G., Frank M., and Kønig H., // Phys. Rev. D56 (1997) 4213;

de Divitiis G.M., Petronzio R., and Silvestrini L., // Nucl. Phys. B504 (1997) 45.

[4] Parke S., FERMILAB-Pub-94/322-T, 1994.

[5] Peccei R.D. and Zhang X. // Nucl. Phys. B337 (1990) 269.

[6] Han T., Peccei R.D., and Zhang X. // Nucl. Phys. B454 (1995) 527.

[7] Han T., Whisnant K., Young B.L., and and Zhang X. // Phys. Lett. B385 (1996) 311.

[8] Hosch M., Whisnant K., and Young B.-L., // Phys. Rev. D56 (1997) 5725 .

[9] Tait T. and Yuan C.-P., MSUHEP-71015, ANL-HEP-PR-97-85, hepph/9710372, 1997.

[10] Obraztsov V.F., Slabospitsky S.R., and Yushchenko O.P., Phys. Lett. B426 (1998) 393.

[11] Arbuzov B.A. and Osipov M.Yu., hep-ph/9802392, 1998.

[12] Han T., Hosch M., Whisnant K., Young B.L., and and Zhang X. // Phys. Rev. D58 (in press), hep-ph/9806486, 1998.

[13] CDF Collaboration, Abe, F. et al., // Phys. Rev. Lett. 80 (1998) 2526. 
[14] CDF Collaboration, Abe, F. et al., // Phys. Rev. Lett. 80 (1998) 2779.

[15] DØ Collaboration, Abachi S. et al., // Phys. Rev. Lett. 79 (1997) 1197;

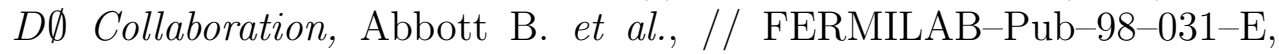
1998.

[16] DØ Collaboration, Klima B., FERMILAB-Conf-98-137-E, 1998. // CDF Collaboration, Velev G.V., FERMILAB-Conf-98-192-E, 1998.

[17] Laenen E., Smith J., and van Neerven W.L., // Phys. Lett. B321 (1994) 254;

Berger E.L. and Contopanagos H. // Phys. Lett. B361 (1995) 115, Phys. Rev. D54 (1996) 3085;

Catani S., Mangano M.L., Nason P., and Trentadue L., // Phys. Lett. B378 (1996) 329. 
Table 1. The values of the anomalous couplings and corresponding branching fractions for top quark decay, calculated within SM framework and by using the values resulting from the Tevatron Run 1 data and from low-energy data. The mass of the top quark is $m_{t}=175 \mathrm{GeV}, \kappa / \Lambda$ is in $\mathrm{TeV}^{-1}$.

\begin{tabular}{|l|c|c|c|c|c|}
\hline & channel & $W^{+} b$ & $q g$ & $q \gamma$ & $q Z$ \\
\hline $\mathrm{SM}$ & $\kappa / \Lambda$ & & $8 \cdot 10^{-6}$ & $3 \cdot 10^{-6}$ & $\kappa_{z} \simeq 4 \cdot 10^{-7}$ \\
\hline & $\mathrm{Br}$ & 1. & $5 \cdot 10^{-11}$ & $5 \cdot 10^{-13}$ & $1.5 \cdot 10^{-13}$ \\
\hline \hline & $\kappa / \Lambda$ & & 1 & 1 & $\kappa_{z}=1$ \\
\hline & $\mathrm{Br}$ & 0.35 & 0.32 & $0.02(0.05)$ & 0.32 \\
\hline \hline FNAL & $\kappa / \Lambda$ & & 0.5 & 0.77 & $\kappa_{z}=0.74$ \\
\hline & $\mathrm{Br}$ & 0.57 & 0.13 & 0.018 & 0.28 \\
\hline \hline Low-energy & $\kappa / \Lambda$ & & 0.5 & 0.28 & $\kappa_{z}=0.29$ \\
\hline & $\mathrm{Br}$ & 0.77 & 0.17 & 0.003 & 0.056 \\
\hline
\end{tabular}

Table 2. Result of the application of differential cuts for the processes of $t t$ production and relevant background reactions (see Section 7 for detail explanation).

\begin{tabular}{|l|c|c|c|}
\hline & signal $t t$ & Bckg. $W t \bar{t}$ & Bckg. $q W q W$ \\
\hline \hline cross section (for $\kappa=1)$ & $0.67 \mathrm{pb}$ & $0.01 \mathrm{pb}$ & $0.01 \mathrm{pb}$ \\
\hline \hline cut & $N_{\text {events }}(t t)$ & $N_{\text {events }}(W t \bar{t})$ & $N_{\text {events }}(q W q W)$ \\
\hline \hline no cut & 10000 & 10000 & 10000 \\
\hline "standard" cuts & 3452 & 5354 & 5462 \\
\hline "two jets" & 2095 & 712 & 3269 \\
\hline " $E_{\top} "$ & 1221 & 316 & 118 \\
\hline$M(l j)<160 \mathrm{GeV}$ & 1177 & 190 & 46 \\
\hline$M(l l j j)>500 \mathrm{GeV}$ & 853 & 15 & 12 \\
\hline \hline$N_{\text {events }}$ for $L=10^{5} \mathrm{pb}^{-1}$ & 5715 & 1.5 & 1.2 \\
\hline
\end{tabular}


a) SM

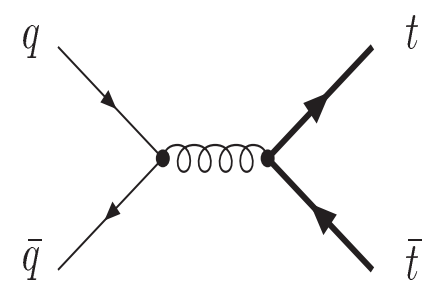

b) FCNC

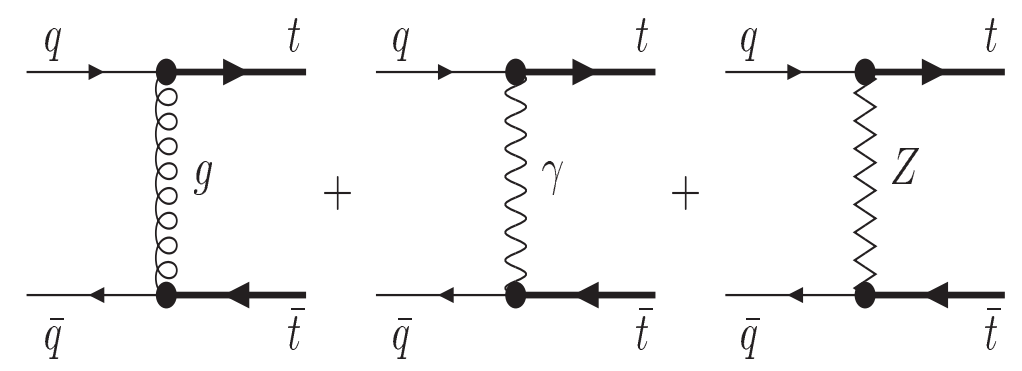

c) SM

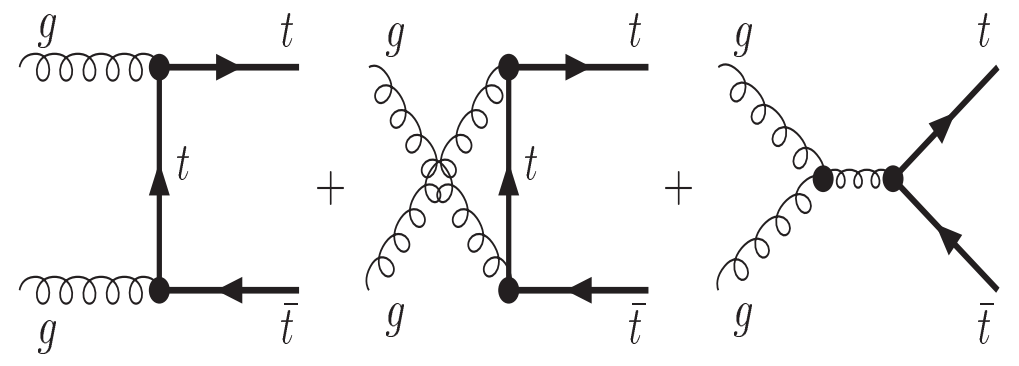

d) FCNC

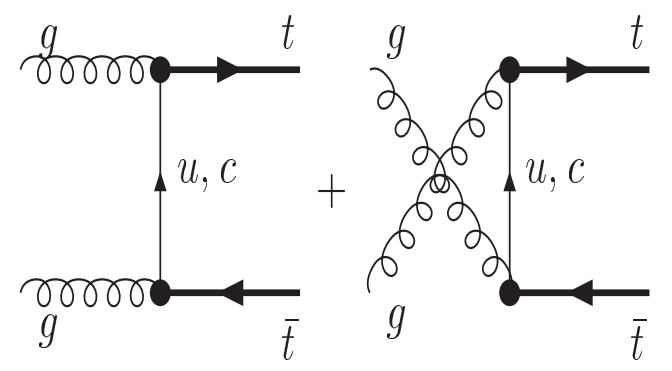

Figure 1: Lowest order diagrams describing $t \bar{t}$ production in QCD (a and $c)$ and in FCNC (b and d). 


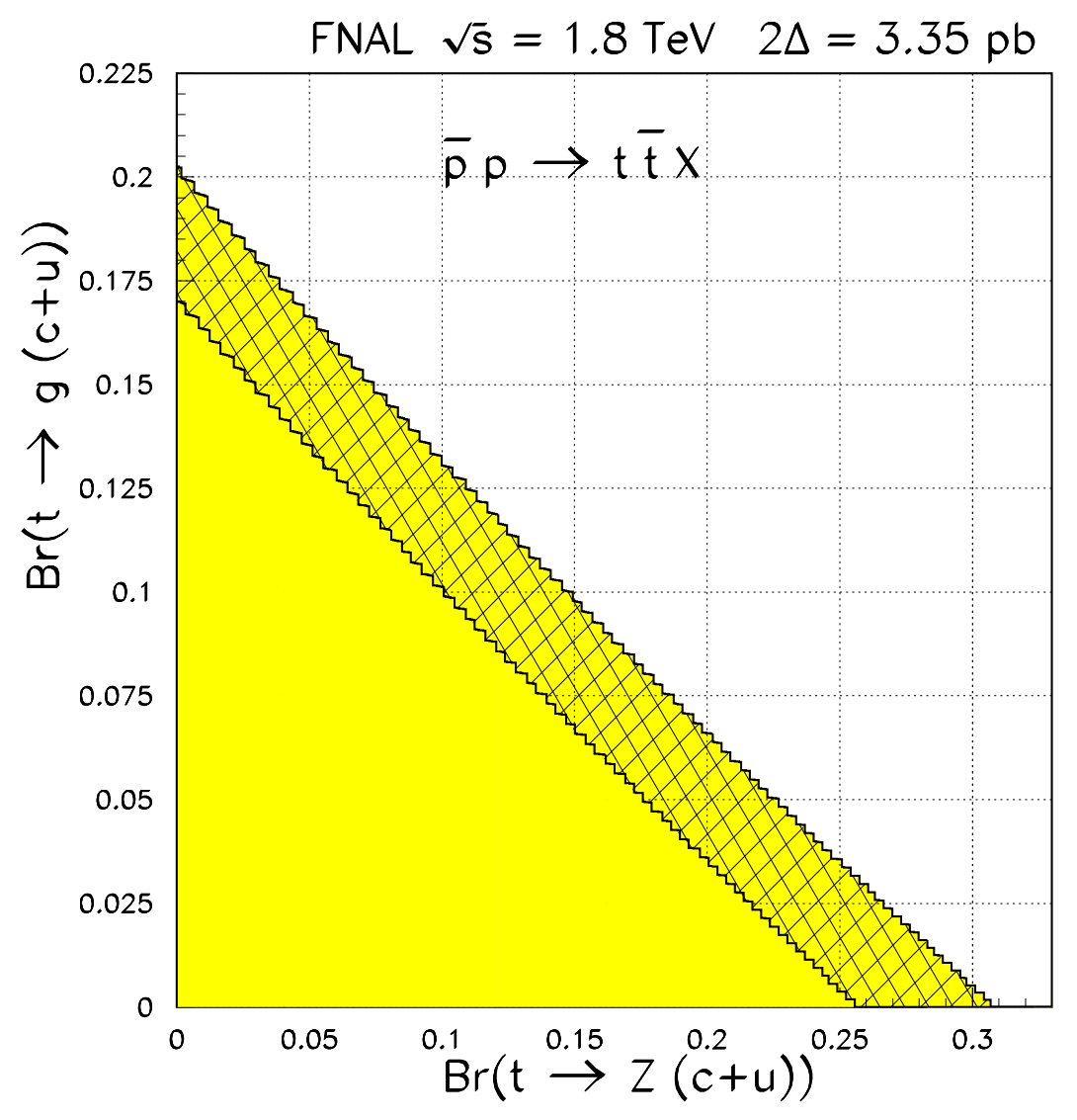

Figure 2: $\quad$ Upper limit on $\operatorname{Br}(t \rightarrow g(c+u))$ versus $\operatorname{Br}(t \rightarrow Z(c+$ $u$ ) resulted from the analysis of the $t \bar{t}$ production at Run 1 of the Tevatron. 

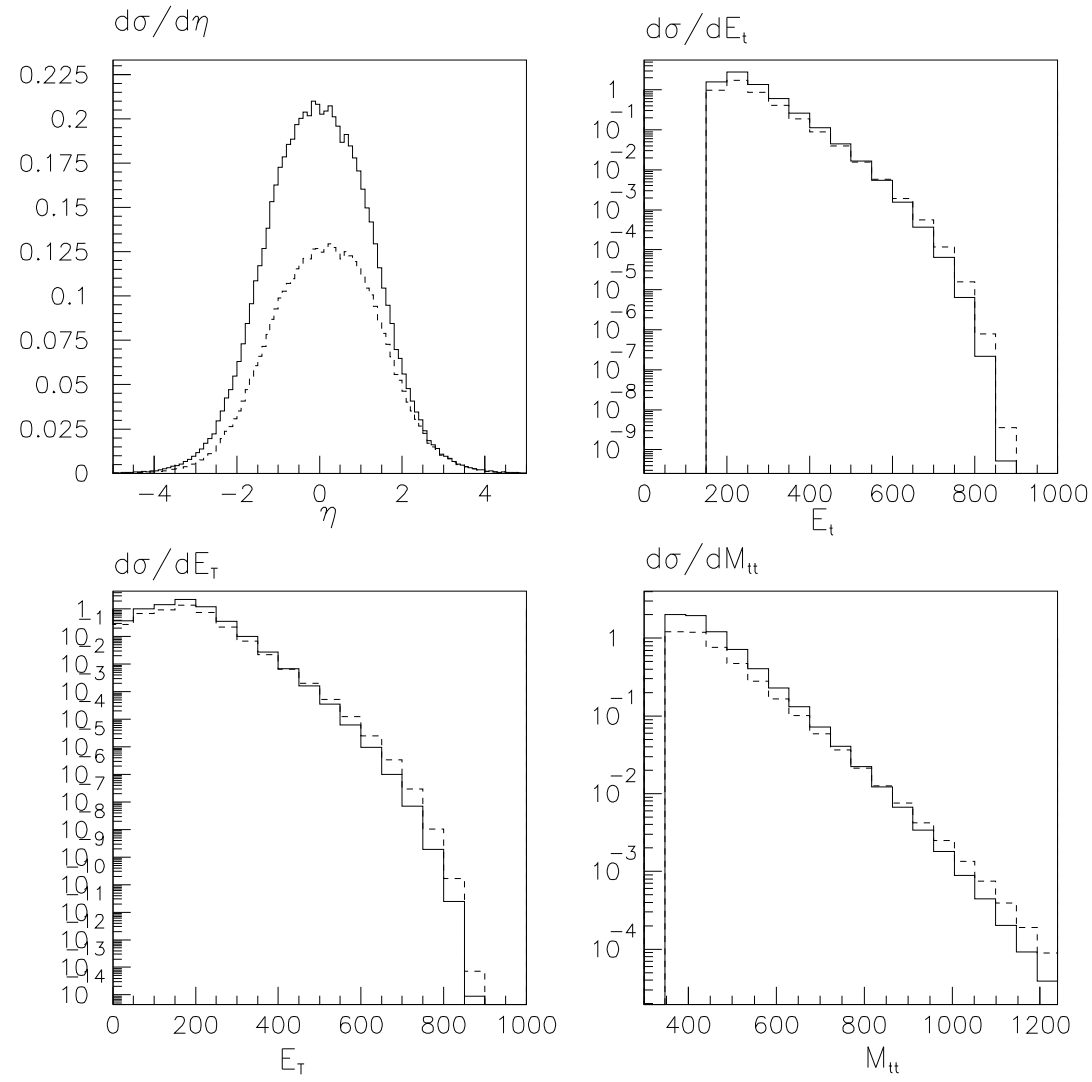

Figure 3: Differential distributions over pseudorapidity $(\eta), t$ quark energy $\left(E_{t}\right)$, transverse energy of the top $\left(E_{T}\right)$, and invariant mass of the $t \bar{t}$ pair $\left(M_{t t}\right)$, for $t$ quark produced in the reaction $\bar{p} p \rightarrow t \bar{t} X$ at $\sqrt{s}=1.8 \mathrm{TeV}$ (FNAL collider). Solid curves are QCD predictions, dashed curves are QCD+FCNC calculations. All distributions are given in arbitrary units. 

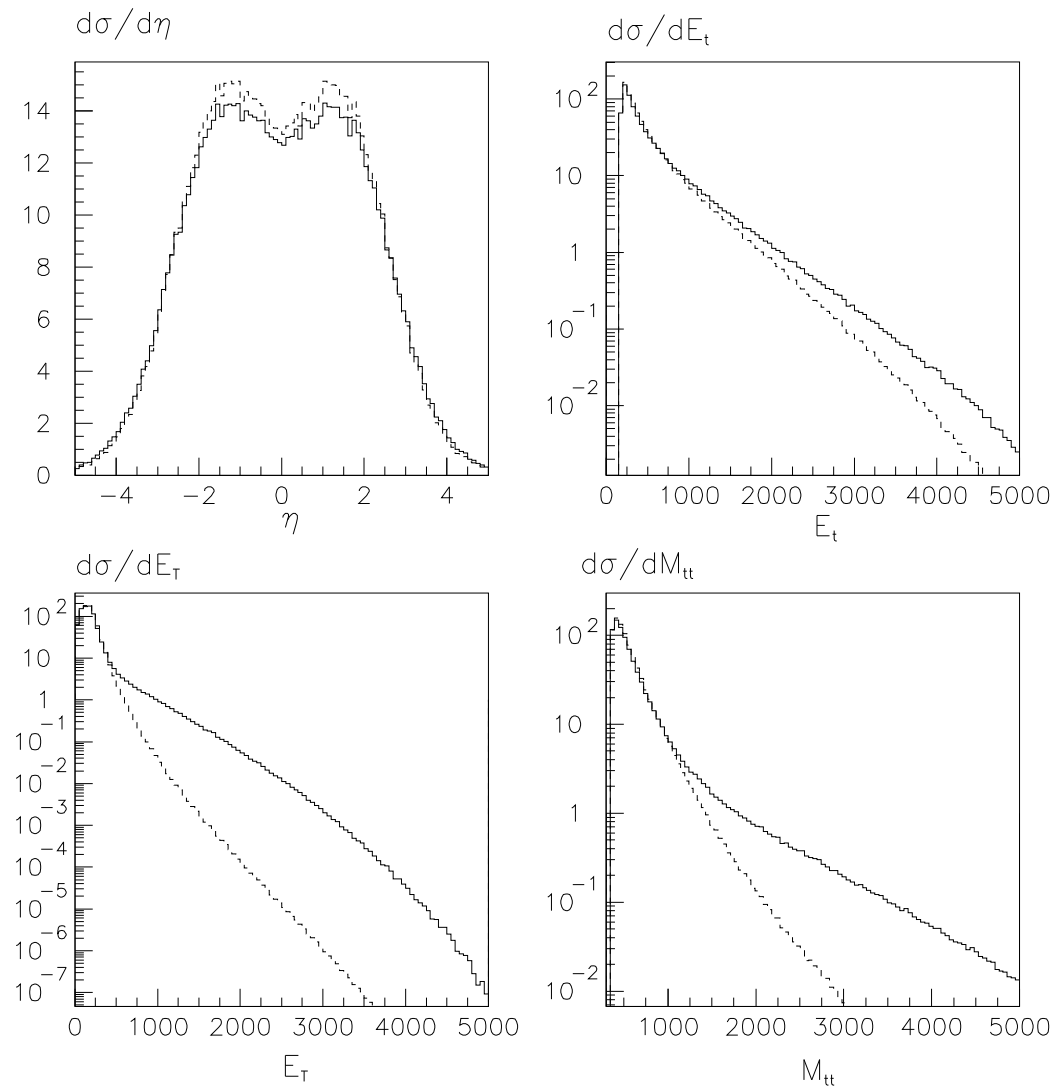

Figure 4: The same distributions as in Fig.3, but calculated for the reaction $p p \rightarrow t \bar{t} X$ at $\sqrt{s}=14 \mathrm{TeV}$ (LHC collider). 


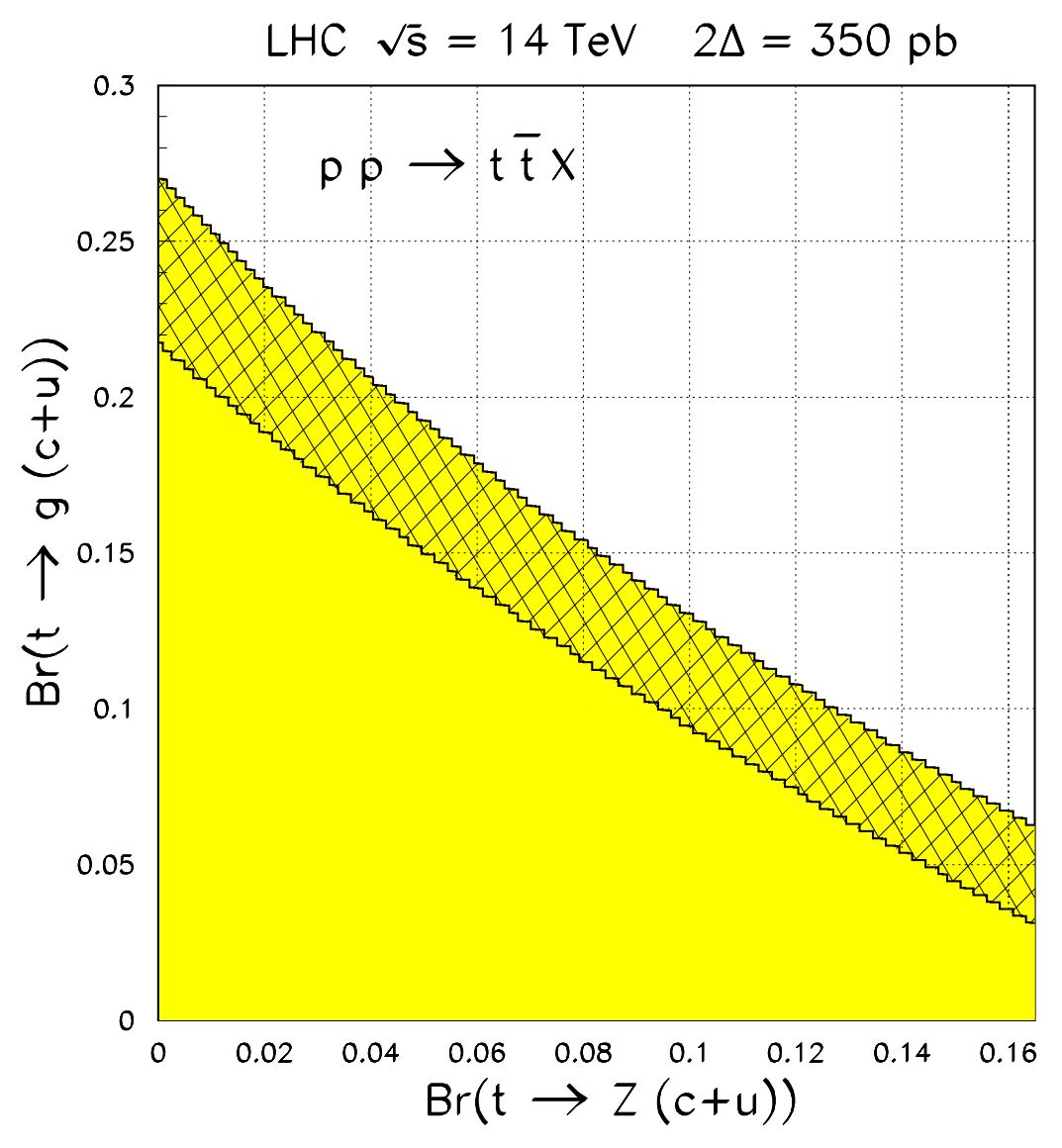

Figure 5: Upper limit on $\operatorname{Br}(t \rightarrow g(c+u))$ versus $\operatorname{Br}(t \rightarrow Z(c+u))$ which may be obtained at LHC collider from analysis of the $t \bar{t}$ pair production cross section. 


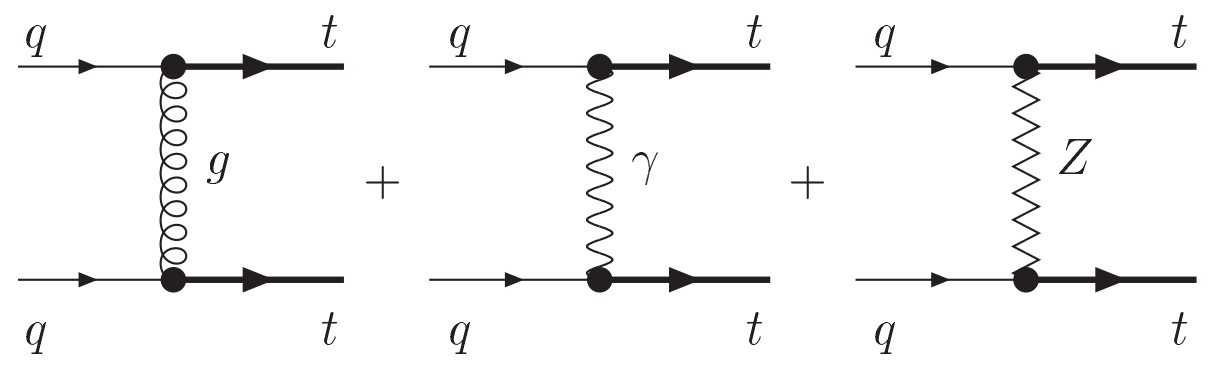

Figure 6: Diagrams describing $t t$ quarks production due to FCNC interaction. 

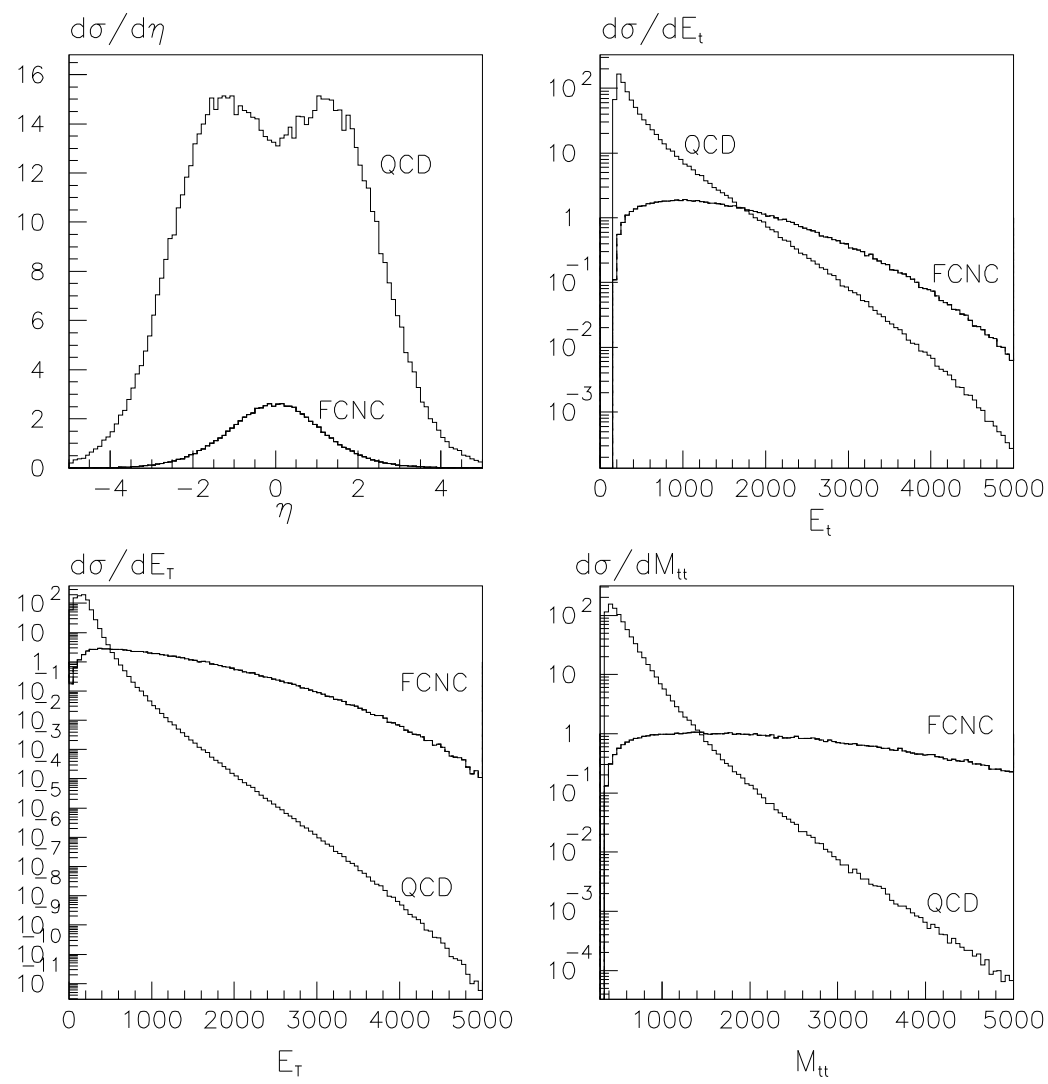

Figure 7: Differential distributions on pseudorapidity $(\eta), t$ quark energy $\left(E_{t}\right)$, transverse energy of the top quark $\left(E_{T}\right)$, and invariant mass of the top-top pair $\left(M_{t t}\right)$, for $t$ quark produced in the reaction $p p \rightarrow t t X$ at $\sqrt{s}=14 \mathrm{TeV}$ (LHC collider). All distributions are given in arbitrary units. (For comparison we show at the same plots the QCD predictions for the case of $t \bar{t}$ production at the same energy). 
a) $q \bar{q} \rightarrow W t \bar{t}$

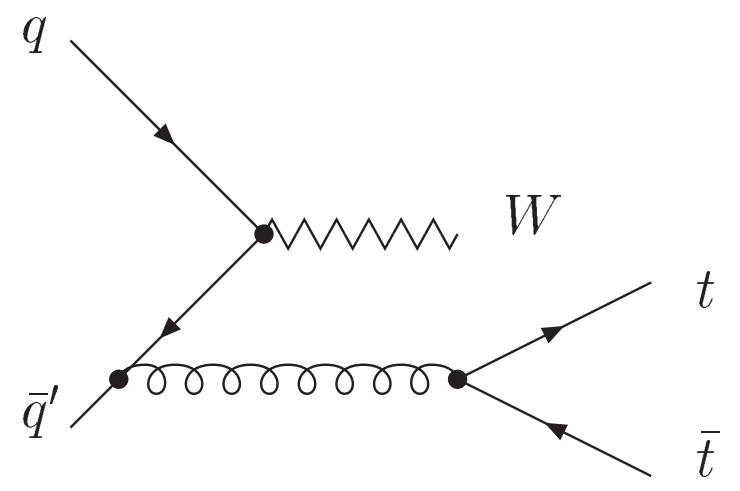

b) $q q \rightarrow W W q q$

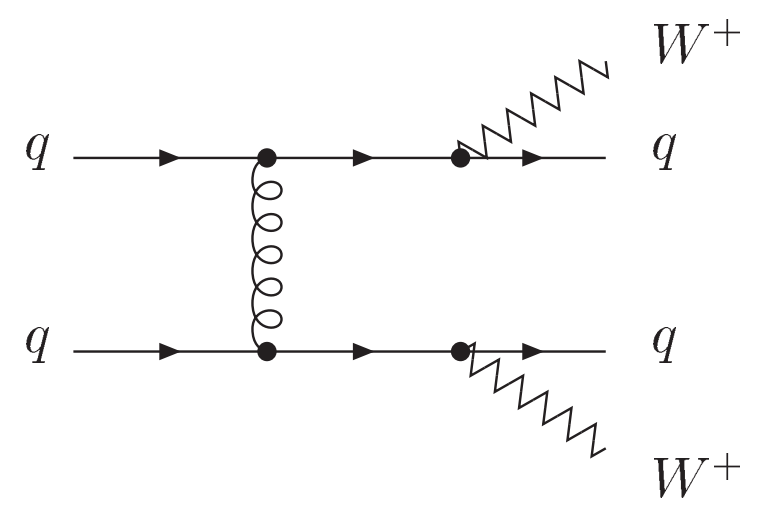

Figure 8: Diagrams describing the background processes for the reaction of $t t$ pair production. 

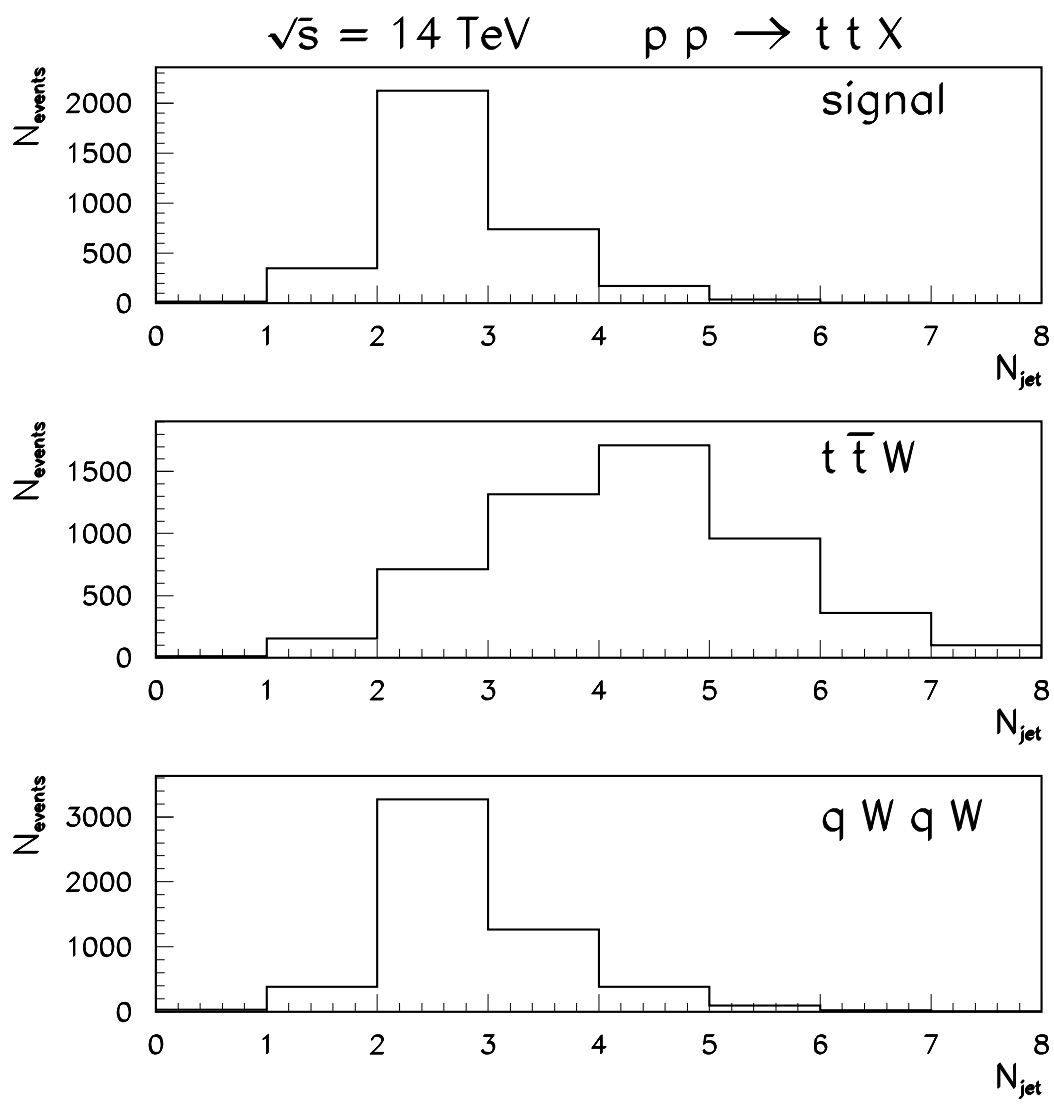

Figure 9: Distributions on number of jets $N_{\text {jet }}$. 

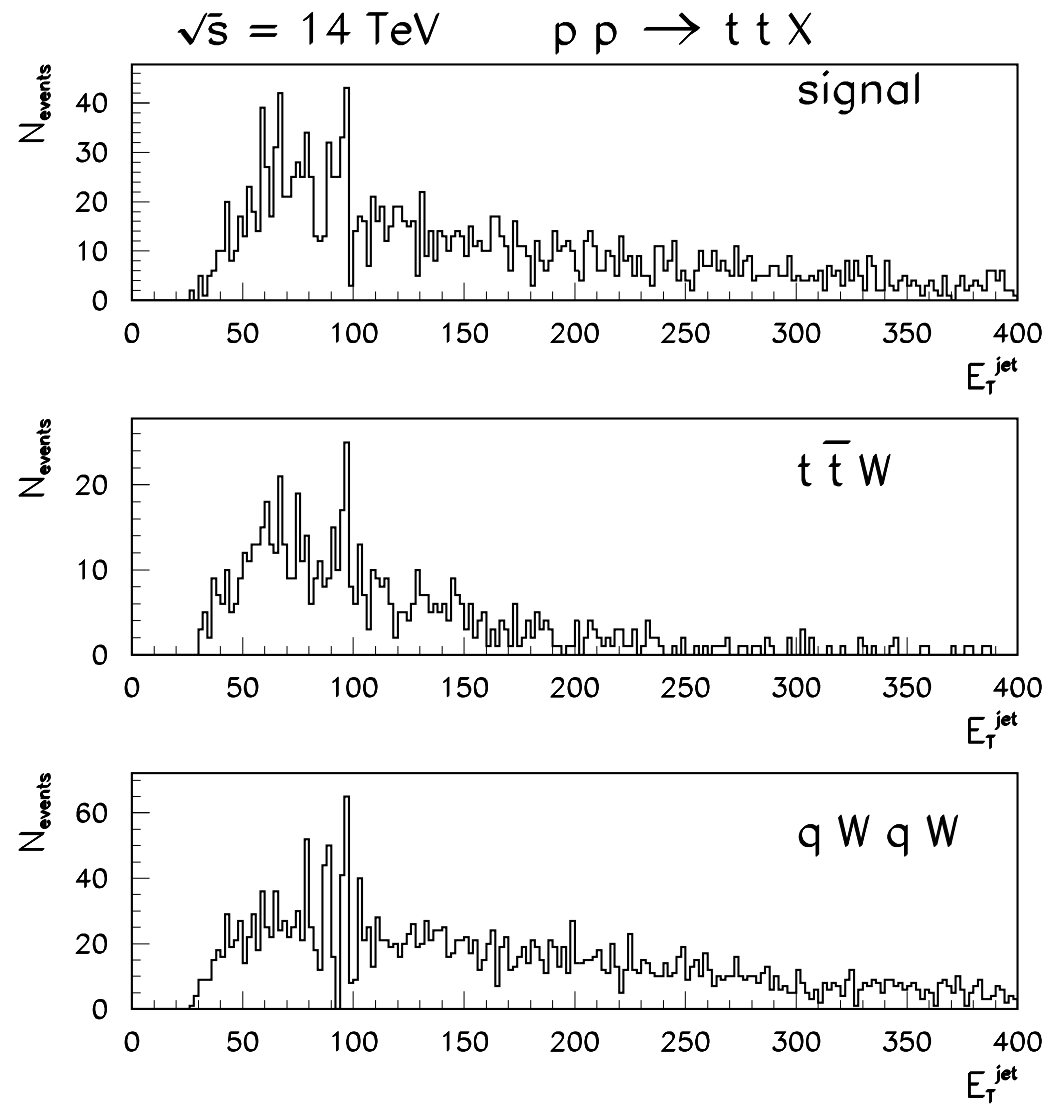

Figure 10: $\quad E_{\top}^{\text {jet }}$-distributions from the signal $(t t)$ and background processes. $E_{T}^{\text {jet }}$ is in $\mathrm{GeV}$. 

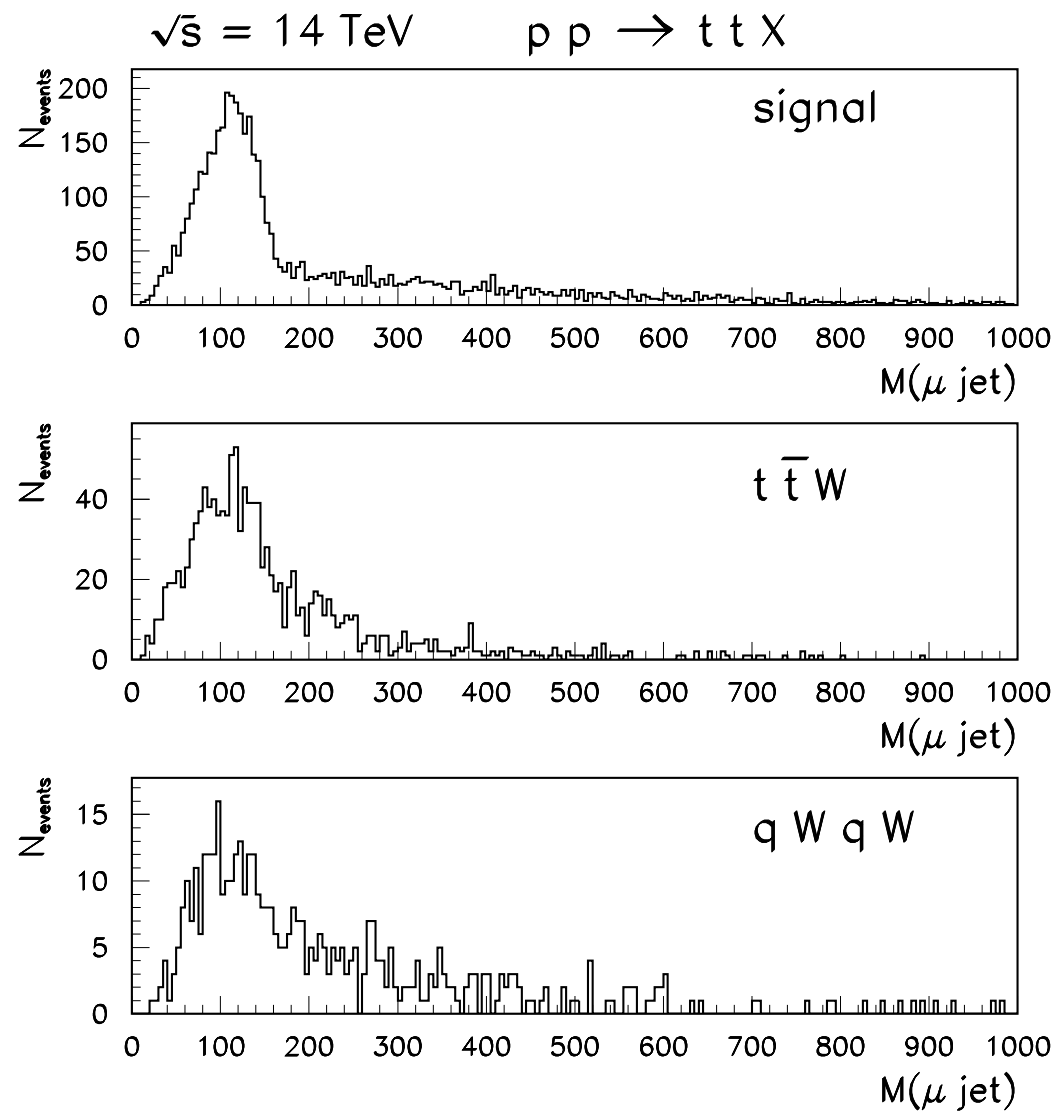

Figure 11: Distributions on invariant mass of charged lepton and jet. All combinations of leptons and jets are taken. $M(\mu$ jet $)$ is in $\mathrm{GeV}$. 

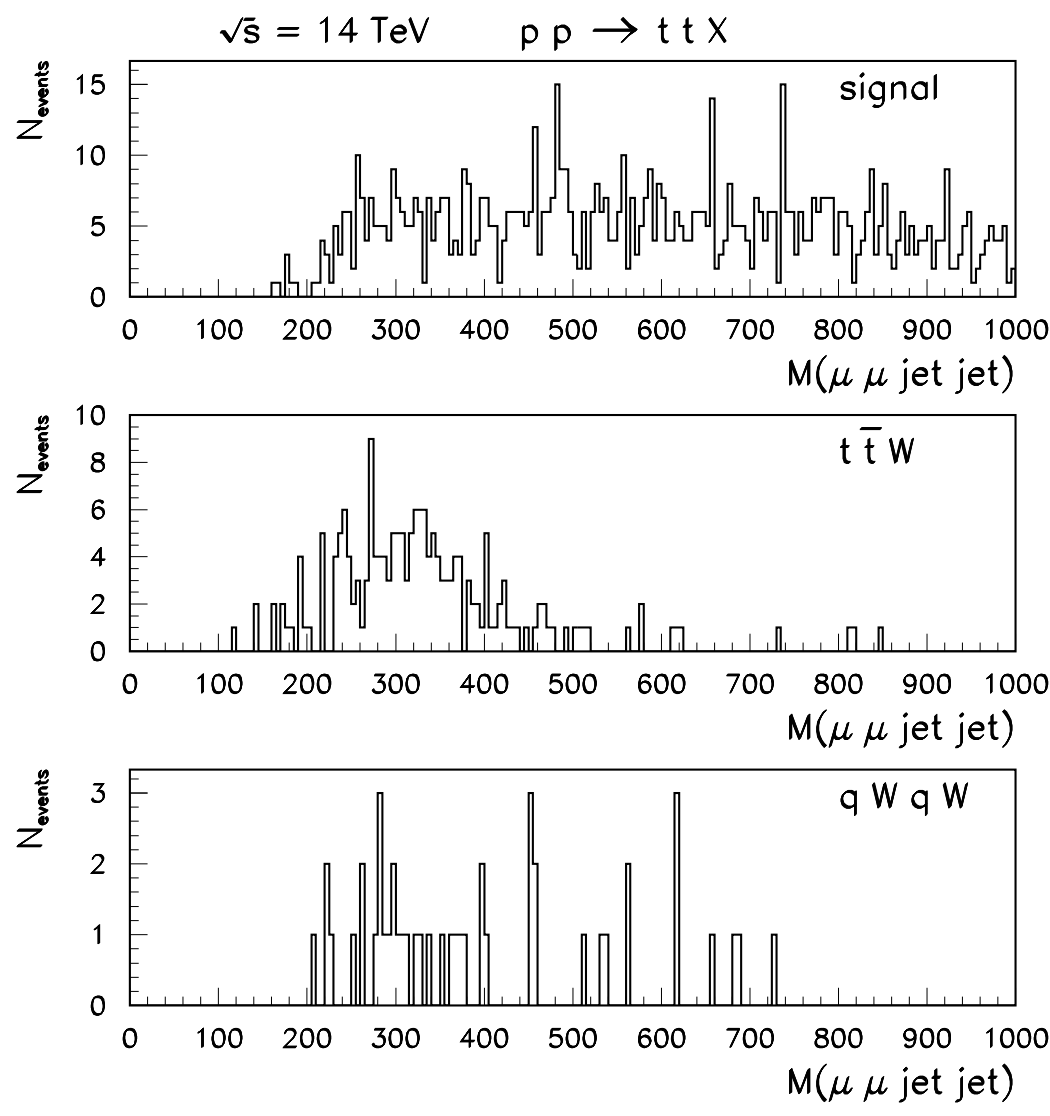

Figure 12: Distributions on invariant mass of two charged leptons and two jets. $M(\mu \mu$ jet jet $)$ is in $\mathrm{GeV}$. 